\title{
The Impact of Infective Immigrants on the Spread of Dog Rabies
}

\author{
Aberu Mengistu Tulu, Purnachandra Rao Koya* \\ School of Mathematical and Statistical Sciences, Hawassa University, Hawassa, Ethiopia \\ Email address: \\ aberumengistu63@gmail.com (A. M. Tulu),drkpraocecc@yahoo.co.in (P. R. Koya) \\ ${ }^{*}$ Corresponding author
}

To cite this article:

Aberu Mengistu Tulu, Purnachandra Rao Koya. The Impact of Infective Immigrants on the Spread of Dog Rabies. American Journal of Applied Mathematics. Vol. 5, No. 3, 2017, pp. 68-77. doi: 10.11648/j.ajam.20170503.12

Received: April 11, 2017; Accepted: May 2, 2017; Published: June 23, 2017

\begin{abstract}
In this paper, it is proposed and analyzed a new mathematical model and that is developed on the basis of some reasonable modification made to the standard epidemic model. The impact of immigration, treatment and the effect of vaccination are included in the model. The basic reproduction number is derived using the next generation matrix method. Disease free equilibrium point is found and endemic equilibrium state is identified. Numerical simulation study is conducted using ode 45 of MATLAB. It has been shown that the solution is positive and bounded. Algebraic expression for the reproduction number is constructed. Equilibrium points are identified and their stability analysis is carried out. It is pointed out that the disease dies out if the immigration of the infected dogs is controlled and the vaccination and the treatments are improved. Otherwise, the disease spreads rapidly in the dog population and it becomes an epidemic. Further, it is also pointed out that the impact of infective immigrants on the spread of dog rabies is positive and additive. The details are presented and discussed in the text.
\end{abstract}

Keywords: Infective Immigrants, Rabies, Vaccination, Compartmental Model, Stability, Reproductive Number, Simulation

\section{Introduction}

In the present world, immigration has become unavoidable activity but it poses a significant risk for disease propagation in every population in general and rabies among dog populations in particular. The effect of immigration on the propagation of diseases will be higher in developing countries which normally do not check the complete health status of immigrants. Rabies is a fatal viral disease caused by the rabies virus. Most countries in both African and Asian continents are identified as high - risk areas for human rabies $[2]$.

The dog rabies disease has been in existence for more than three thousand years and thus it is the oldest infectious disease known to medical science. Rabies virus has abroad mammalian host range. Rabies occurs significantly in more than 150 countries and in territories around the world, and it is very high in developing countries of Africa and Asia. Poor and rural communities are highly vulnerable to rabies due to their interaction with domestic animals including dogs [2 -5].
Rabies virus is transmitted in the form of saliva through the bite of an infected animal. Dog bites are a major cause of rabies in humans, and mass vaccination of dogs could be an effective control measure. Children are the most affected victims. According to the reports about $40 \%$ people bitten by infected or rabid animals are children. This is due to the fact that the children have higher tendency to play with animals. More than 15 million people receive post exposure vaccination worldwide to be protected from the disease [6-8].

Most of the North American and European countries had fought against the rabies disease and were successful in eradicating the virus. Even than about 61 thousand deaths occurred in the year 2010 due to rabies disease world wide as reported by the WHO. Of course about $95 \%$ of these deaths occurred in the African and Asian continents [9-12].

The first known major outbreak of rabies due to dogs occurred in many parts of Ethiopia in the year 1884. The first case of rabies epidemic was reported and had a high prevalence in Addis Ababa, the capital city of Ethiopia. Rabies cases are reported from all regions of Ethiopia, and it has persisted for century and more than 2000 people are 
bitten by dog annually in and around Addis Ababa [13].

Most rabies modeling has focused on disease dynamics and control within terrestrial mammals e.g., raccoons and foxes. Rabies still poses a significant human health problem throughout most of Africa, where the majority of the human rabies cases results from dog bites. World Health Organization WHO statistics indicate that about 55000 human deaths have been occurring worldwide pre dominantly in Africa and Asia. The expenditure on the treatment and control exceeds US \$500 million annually worldwide [14 - 15].

Rabies problem not only relates to dogs but also humans. Basically the success of control and eradication programs of rabies depends on the level of people awareness. There is a necessity for changing of behavior that allows the community to accept various obligations to impound and bind their dogs. Public have to well feed, care and maintain their dogs' health and periodically provide them vaccinations. The rabies can attack at all ages and genders [16].

Dog rabies is estimated to cause 24,000 human deaths per year in Africa, while Africa is the second continent mostly affected by the disease. The main cause of transmission of rabies to human in Africa is by a bite of a rabid dog. About $30 \%$ to $60 \%$ of dog bite victims in dog - endemic areas are children of less than 15 years of age. Dogs are the most important reservoirs for the rabid virus and have been the source of transmitting it to $99 \%$ of all reported human cases [17-18].

Transmission of rabies: Any warm - blooded animals including humans may become infected with the rabies virus and develop symptoms. The virus is transmitted to the victim when virus - laden saliva is introduced through a bite or a scratch from a rabid animal. In nature it is transmitted from animal to animal by means of a bite introducing the saliva bearing the virus. Rarely, rabies may be transmitted by viral contamination of fresh or already existing wounds. Virus may be presented in the saliva and be transmitted by an infected animal several days prior to the onset of clinical signs. Indirect exposure to saliva of a rabid animal can also occur through contact with a pet that has had recent contact with a rabid animal. Broken skin or mucous membrane exposure to nervous tissue e.g., brain and spinal cord of an infected animal may also pose a risk of transmission. After thousands of years of reports on rabies, direct person to person transmission has not been documented. However, there have been cases documented after corneal and solid organ transplants from individuals with unappreciated infection. Droplet transmission e.g., in a cave with a multitude of bats or in a laboratory handling rabies virus, has occurred. Rabies is not transmitted through contact with blood, urine, skunk spray, or feces of an infected animal.

Incubation period: The period from the time from infection to the onset of symptoms is known as the rabies incubation period. During the rabies incubation period, a bite by the infected animal does not carry a risk of rabies because the virus is not yet in the saliva. The incubation period of the disease depends on how far the virus must travel to reach the central nervous system. The incubation period varies but generally from 3 to 7 weeks. During this time, the rabies virus is multiplying within the body. Mainly the disease period is divided in to three stages: Prodromal stage, Excitative stage, and Dumb or Paralytic stage.

Prodromal stage: The prodromal period lasts for 1 to 3 days. At the beginning there will be a change in the behavior of animal and that often may be slight in later times and thus may be overlooked. The symptoms include shyness, nervousness, difficulty in swallowing and sometimes salivation. The animal may also stop eating and drinking and also prefers to be alone and single.

Excitative stage: Excitative stage is also known as raging fury or mad - dog syndrome stage. This stage is characterized by irrational and vicious aggressiveness, restlessness, excitement and mania for biting and snapping. During this stage the saliva is highly infectious. As the disease progresses muscular in coordination and convulsive seizures becomes common. That is, the muscles of the body and legs begin to tremble making it unable to walk steadily and also breathing becomes very difficult. The animals in this stage can be found roaming on streets and on highways, biting other animals, people and any moving objects and swallowing of foreign bodies etc.

Dumb or Paralytic stage: Animals in dumb stage of rabies appear depressed, lethargic and uncoordinated. Gradually they become complete paralyzed. If paralysis is prominent, this stage is also called silent fury. Paralysis progresses to all parts of the body and leads the animal to coma and then to death in a few hours later. This stage occurs and appears shortly before death.

Treatment: The first step in treating a person bitten by any animal is to wash the wound with soap and water. The infection virus dies in dried saliva within a few hours. It is also killed by ordinary sunlight, heat, household detergent and disinfectants. Pure iodine and hydrogen peroxide however have no effect on the virus. If the symptoms begin to show up then there is no cure and death is almost certain, but treatment involves only supportive care. However, if a dog or a person is bitten by a rabid animal and has not yet experienced symptoms, there is an extremely effective post exposure treatment. Most of the time, stitches should not be used for animal bite wounds.

Rabies vaccine: Rabies vaccine works in two ways, either after or before an exposure. A person infected with rabies and does not obtain treatment before occurrence of the symptoms dies in a short period after experiencing convulsions and other violent nervous symptoms. Dogs continue to be the main carrier of rabies in Africa and Asia and are responsible for most of the human rabies deaths worldwide.

Epidemiological models: The earliest account of the mathematical modeling of the spread of a disease was carried out in 1766 by Daniel Bernoulli. Trained as a physician, Bernoulli created a mathematical model to defend the practice of inoculating against smallpox. The calculations from this model showed that universal inoculation against smallpox would increase the life expectancy from 26 years 7 
months to 29 years 9 months. Inoculation is a kind of treatment of a disease and is done by sending in a vaccine into the blood [8].

Early models of rabies dynamics were similar to early models for most other diseases and followed the basic $S E I R$ frame work. In these models populations are sub divided into specific classes corresponding to susceptible $S$, exposed $E$, infectious $I$, and recovered or removed $R$ individuals. The dynamics are included through the construction of a system of ordinary differential equations. These models represent either a single population or linked populations. From the mathematical analysis of these models a variety of predictions have been drawn concerning temporal and spatial patterns [4].

Although these foundational SEIR models may have lacked the level of mathematical sophistication, there is really no need for highly complicated mathematical representations since the availability and reliability of data were rather limited. For instance, some recent models for describing rabies dynamics incorporate explicit spatial interactions and account for events that are discrete in temporal and special coordinates. These spatially explicit models may not have provided much improvement over the earliest ODE models used 30 years ago, because early in the epidemic, detailed temporal history was not yet available and spatial resolution was limited to densities of individuals within large regions [4].

\section{Mathematical Modeling of Rabies}

The mathematical model is formulated using ordinary differential equations. Simulation of the model is conducted using the computer software MATLAB.

\subsection{Model Formulation}

In this study the existing standard SEIR model has been modified and constructed an improved and extended model called as $S E I_{1} I_{2} R$. For the standard $S E I R$ model, the whole dog populations categorized into four classes: (i) Susceptible class $S$ refers to the healthy dogs that have not yet caught the rabies virus but are likely to contract the disease (ii) Exposed class $E$ refers to the dogs that have been bitten by infected dogs but are not made up to be infectious (iii) Infective class $I$ refers to the dogs infected with rabies virus and are contagious make up and (iv) the removed class $R$ constitute dogs which have died from the infection.

In the modified model $S E I_{1} I_{2} R$, dog population is categorized into five classes. The notations $S$ and $E$ represent susceptible and exposed classes $I_{1}$ represent infected but prodromal stage and $I_{2}$ represent infected furious stage and $R$ represent recovery class.

\subsection{SEIR Model Without Vaccination}

In the standard SEIR model, the population is divided into four compartments. Dog population in the susceptible compartment is neither exposed nor infected by rabies disease. But they are very sensitive or easily influenced and likely to be affected by or having the chance of receiving the disease in future. Dogs that have been bitten by infected ones but are not infectious make up the exposed class. Dogs that are infected with rabies virus and contagious make up the infective class. The removed class constitutes dogs which have died of the infection. The proportions of individuals in the compartments $S, E, I, R$, at time $t$, are denoted by $S(t), E(t), I(t)$ and $R(t)$ respectively.

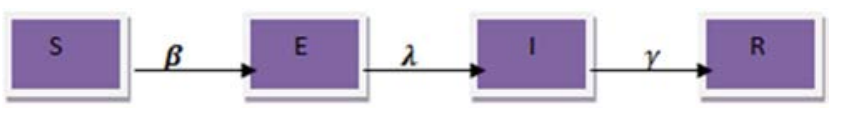

Figure 1. Flow diagram of the SEIR model.

The SEIR model can be represented by a system of ordinary differential equations mentioned as follows:

$$
\begin{gathered}
d S / d t=-\beta S(I / N) \\
d E / d t=\beta S(I / N)-\lambda \mathrm{E} \\
d I / d t=\lambda \mathrm{E}-\gamma I \\
d S / d t=\gamma I
\end{gathered}
$$

The total population $N$ sum of populations of all the four compartments $N=S+E+I+R$. Here in (1) to (4) the notations used include: $\beta$ is the transmission coefficient of rabies between $\operatorname{dogs}, \lambda$ is the latency or incubation rate in dogs and $\gamma$ is the death rate of dogs due to disease.

\subsection{Assumptions of the Proposed Model with Vaccination}

The proposed and modified $S E I_{1} I_{2} R$ model can be applied on dog rabies and is used compute the population sizes and dynamics of susceptible, exposed, infected, recovered due to vaccination and removed populations. The model constructed with the consideration of the following assumptions:

(i) The immigration is considered. Hence, the dog population under consideration is heterogeneous and varying with time.

(ii) The $R$ compartment constitutes dogs which have recovered from the infection up on administration of the rabies vaccine. This will be called as the recovered class $R$.

(iii) A portion $\theta S$ of the susceptible population goes to the recovered class $R$ directly due to pre-exposure vaccination.

(iv) A portion $\theta E$ of the exposed population goes to the recovered class $R$ directly due to post-exposure vaccination.

(v) A portion $\delta R$ of the recovered population goes to the susceptible class $S$ directly due to the waning immunity of the rabies vaccine.

\subsection{Mathematical Modeling of the Proposed Model with Vaccination}

The compartmental structure of the model and flow directions and rates of populations among the compartments are shown in the form of a flow diagram as follows: 


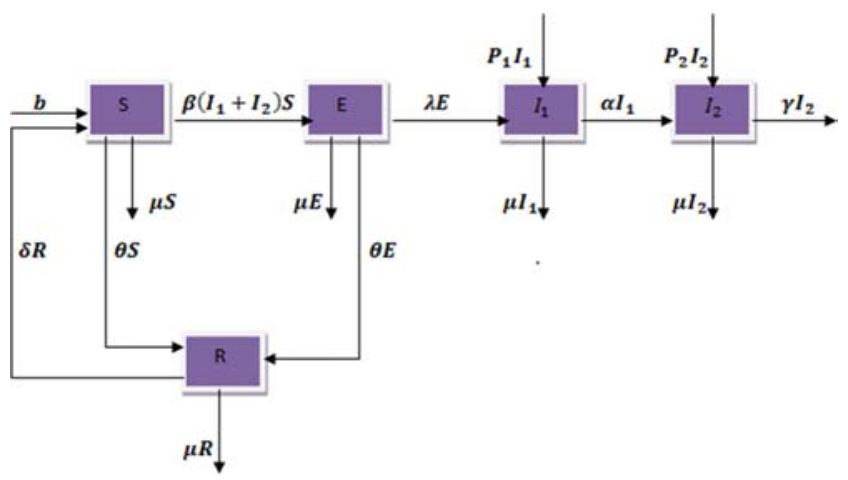

Figure 2. Flow diagram of the proposed $S E I_{1} I_{2} R$ model.

The system of ODE equations representing the $S E I_{1} I_{2} R$ model can be constructed based on the flow diagram as follows:

$$
\begin{gathered}
d S / d t=b-\beta\left(I_{1}+I_{2}\right) S / N-(\theta+\mu) S+\delta R \\
d E / d t=\beta\left(I_{1}+I_{2}\right) S / N-(\lambda+\theta+\mu) E \\
d I_{1} / d t=\lambda E+P_{1} \mathrm{I}_{1}-(\alpha+\mu) I_{1} \\
d I_{2} / d t=\alpha I_{1}+P_{2} \mathrm{I}_{2}-(\gamma+\mu) \mathrm{I}_{2} \\
d R / d t=\theta S+\theta E-(\delta+\mu) R
\end{gathered}
$$

The total population is the sum of the populations of all the five compartments i.e., $N=S+E+I_{1}+I_{2}+R$.

The symbolic notations of the variables and their descriptions: $S(t)$ is susceptible dog population at time $t$; $E(t)$ is exposed dog population at time $t ; I_{1}(t)$ is infectious or prodromal stage dog population at time $t ; I_{2}(t)$ is Infectious or furious stage dog population at time $t ; R(t)$ is recovered dog population at time $t$.

The symbolic notations of the parameters and their descriptions: $\beta$ is transmission coefficient of the disease among dogs; $\lambda$ is latency or incubation rate of the disease in dogs; $\gamma$ is death rate of dogs due to disease; $\theta$ is vaccination rate coefficient; $\delta$ is the loss rate of vaccination immunity for dogs; $\mu$ is natural death rate of dogs; $\alpha$ is rate of propagation of furiousness among dogs; $b$ is birth rate of $\operatorname{dogs} ; P_{1}$ is immigration to prodromal stage $\operatorname{dog}$ population; and $P_{2}$ is immigration to furious stage dog population.

\subsection{Positivity of the Solutions of the Modified Model}

In order to show that the general model equations (5) to (9) are to be epidemiologically meaningful and well posed, it is needed to prove that all the state variables are non - negative. This fact has been stated as a theorem and proved in what follows.

Theorem 1 If the initial conditions are non - negative i.e., $S(0)>0, E(0)>0, I_{1}(0) \geq 0, I_{2} \geq(0), R>0$ then all the solutions $\left\{S(t), E(t), I_{1}(t), I_{2}(t), R(t)\right\}$ of the system of the equations (5) - (9) are also non - negative for all $t>0$. Proof To show that the solution of the dynamical system comprising of the equations (5) to (9) is positive it is needed to consider and verify each differential equation and show that their solutions are positive.
Firstly, the differential equation (5) $d S / d t=b-$ $\left\{\left[\beta\left(I_{1}+I_{2}\right) S\right] / N\right\}-(\theta+\mu) S+\delta R$ can be expressed without loss of generality as an inequality as $d S / d t>$ $-\left\{\left[\beta\left(I_{1}+I_{2}\right) S\right] / N\right\}-(\theta+\mu) S$. Up on integrating the inequality the analytic solution is obtained as $S(t)>$ $S(0) \mathrm{e}^{-\left\{\left\{\left[\beta\left(I_{1}+I_{2}\right)\right] / N\right\}-(\theta+\mu)\right\} t}$. Here $S(0)$ is integral constant and represents initial population of the susceptible compartment and hence it is a positive quantity. Now within the limit $t \rightarrow \infty$ the analytical solution leads to $S(t)>0$. Hence the solution or the population size of the susceptible compartment $S(t)$ is always positive.

Secondly, the differential equation (6) $d E / d t=$ $\beta\left(I_{1}+I_{2}\right) S / N-(\lambda+\theta+\mu) E$ can be expressed without loss of generality as an inequality $d E / E \geq-(\lambda+\theta+$ $\mu) d t$. Up on integrating the inequality an analytic solution is obtained as $E(t) \geq E(0) e^{-(\lambda+\theta+\mu) t}$. Here $E(0)$ is integral constant and represents initial population of the exposed compartment and hence it is a positive quantity. Now within the limit $t \rightarrow \infty$ the analytical solution leads to $E(t) \geq 0$. Hence the solution or the population size of the exposed compartment $E(t)$ is always non-negative.

Thirdly, the differential equation (7) $d I_{1} / d t=\lambda E+$ $P_{1} I_{1}-(\alpha+\mu) I_{1}$ can be expressed without loss of generality as an inequality $d I_{1} / d t \geq-(\alpha+\mu) \mathrm{dt}$. Up on integrating the inequality an analytic solution is obtained as $I_{1}(t) \geq$ $I_{1}(0) e^{-(\alpha+\mu) t}$. Here $I_{1}(0)$ is integral constant and represent initial population of the prodromal stage infected compartment and hence it is a positive quantity. Now within the limit $t \rightarrow \infty$ the analytical solution leads to $I_{1}(t) \geq 0$. Hence the solution or the population size of the prodromal stage infected compartment $I_{1}(t)$ is always non-negative.

Fourthly, the differential equation (8) $d I_{2} / d t=\alpha I_{1}+$ $P_{2} I_{2}-(\gamma+\mu) I_{2}$ can be expressed without loss of generality as an inequality $d I_{1} / I_{2} \geq-(\gamma+\mu) d t$. Up on integrating the inequality an analytic solution is obtained as $I_{2}(t) \geq I_{2}(0) e^{-(\gamma+\mu) t}$. Here $I_{2}(0)$ is integral constant and represent initial population of the furious stage compartment and hence it is a positive quantity. Now within the limit $t \rightarrow \infty$ the analytical solution leads to $I_{2}(t) \geq 0$. Hence the solution or the population size of the furious stage infected compartment $I_{2}(t)$ is always non-negative.

Finally, the differential equation (9) $d R / d t=\theta S+\theta E-$ $(\delta+\mu) R$ can be expressed without loss of generality as an inequality $d R / R \geq-(\delta+\mu) d t$. Up on integrating the inequality an analytic solution is obtained as $R(t) \geq$ $R(0) e^{-(\delta+\mu) t}$. Here $R(0)$ is integral constant and represent initial population of the recovery compartment and hence it is a positive quantity. Now within the limit $t \rightarrow \infty$ the analytical solution leads to $R(t) \geq 0$. Hence the solution or the population size of the recovery compartment $R(t)$ is always non-negative.

Therefore, the solution set $\left\{S(t), E(t), I_{1}(t), I_{2}(t), R(t)\right\}$ of equations (5) to (9) are all non-negative for all $t \geq 0$.

\subsection{Boundedness of Solutions of the Modified Model}

In order to show that the solution of general model equations (5) to (9) is bounded it is needed to prove that the total population size $N(t)$ is bounded. This fact is stated in 
the form of a theorem below accompanied by the proof.

Theorem 2 All the solutions $\left\{S(t), E(t), I_{1}(t), I_{2}(t), R(t)\right\}$ of the system of the equations $(5)-(9)$ are bounded.

Proof In order to show that the population sizes of each compartment are bounded it is preferred to show that the total population size of the whole system $N(t)$ is bounded.

The total dog population size $N(t)$ of the whole system is the sum of the populations of the five compartments and is given by $N(t)=S(t)+E(t)+I_{1}(t)+I_{2}(t)+R(t)$. Up on differentiating with respect to time it is obtained $\quad(d N / d t)=(d S / d t)+(d E / d t)+\left(d I_{1} / d t\right)+$ $\left(d I_{2} / d t\right)+(d R / d t)$. Making the use of the equations (5)(9) the expression for $(d N / d t)$ takes the form as $d N / d t=$ $b-\mu N-\gamma I_{2}+P_{1} I_{1}+P_{2} I_{2}$. Note that here $\gamma I_{1} \leq 0$ and hence without loss of generality it can be expressed as $d N / d t=b-\mu N-\gamma I_{2}+P_{1} I_{1}+P_{2} I_{2} \geq b-\mu N \quad$ or equivalently $d N /(b-\mu N) \geq d t$. On integrating the foregoing differential inequality on both sides and applying the initial conditions its analytical solution is obtained as $N(t) \leq(b / \mu)+\{N(0)-(b / \mu)\} e^{-\mu t}$. Now, within the limit $t \rightarrow \infty$ the analytical solution leads to $N(t) \leq b / \mu$. This implies that the upper boundary for the total population size $N(t)$ is $(b / \mu)$.

Therefore, any solution to the system of equations (5) - (9) is bounded i.e., $0 \leq\left\{S, E, I_{1}, I_{2}, R\right\} \leq b / \mu$.

\subsection{Basic Reproductive Number}

The basic reproduction number $R_{0}$ is a threshold parameter defined as the average number of secondary infections caused by an infectious individual when introduced into a completely susceptible population. It is also called basic reproduction ratio or basic reproductive rate. If more than one secondary infection is produced from one primary infection that is, $R_{0}>1$ then an epidemic occurs. When $R_{0}<1$ then there is no epidemic and it means that the disease dies out over a period of time. When $R_{0}=1$ then the disease becomes endemic, meaning the disease remains in the population at a constant rate as one infected dog transmits the disease to one susceptible [9].

Using the next generation matrix approach $R_{0}$ can calculated. The matrix is a composition of two matrices $F$ and $V$. The elements in matrix $F$ constitute the new infections those will arise, while that of matrix $V$ constitutes the transfer of infections from one compartment to another. Here $R_{0}$ is a dominant eigenvalue of the matrix $F V^{-1}$.

The infected compartments are $E, I_{1}$, and $I_{2}$. The rates of changes of populations of these compartments are given by $(6),(7)$ and (8). From the system (6) - (8), the quantities $f_{i}$ and $v_{i}$ are defined as

$$
f_{i}=\left[\begin{array}{c}
\frac{\beta\left(I_{1}+I_{2}\right) S}{N} \\
0 \\
0
\end{array}\right] v_{i}=\left[\begin{array}{c}
(\lambda+\theta+\mu) E \\
(\alpha+\mu) I_{1}-\lambda E-P_{1} I_{1} \\
(\gamma+\mu) I_{2}-\alpha I_{1}-P_{2} I_{2}
\end{array}\right]
$$

Finding the partial derivatives with respect to $E, I_{1}, I_{2}$ and evaluating them at the disease free equilibrium point gives the Jacobian matrices as

$$
\begin{gathered}
F=\left[\begin{array}{ccc}
0 & \beta & \beta \\
0 & 0 & 0 \\
0 & 0 & 0
\end{array}\right] \\
V=\left[\begin{array}{ccc}
\lambda+\theta+\mu & 0 & 0 \\
-\lambda & \alpha+\mu-P_{1} & 0 \\
0 & -\alpha & \gamma+\mu-P_{2}
\end{array}\right]
\end{gathered}
$$

Here the fact used is $(S / N)=1$ which holds good at the disease free equilibrium point $E_{0}$. Now $V$ a lower triangular matrix and hence its determinant is the product of main diagonal elements. Hence, $\operatorname{det}(V)=(\lambda+\theta+\mu)(\alpha+\mu-$ $\left.P_{1}\right)\left(\gamma+\mu-P_{2}\right)$. After some algebraic computation the inverse of $V$ given as

$$
V^{-1}=\left[\begin{array}{ccc}
\frac{1}{\lambda+\theta+\mu} & 0 & 0 \\
\frac{\lambda}{(\lambda+\theta+\mu)\left(\alpha+\mu-P_{1}\right)} & \frac{1}{\alpha+\mu-P_{1}} & 0 \\
\frac{\lambda \alpha}{(\lambda+\theta+\mu)\left(\alpha+\mu-P_{1}\right)\left(\gamma+\mu-P_{2}\right)} & \frac{\alpha}{\left(\alpha+\mu-P_{1}\right)\left(\gamma+\mu-P_{2}\right)} & \frac{1}{\left(\gamma+\mu-P_{2}\right)}
\end{array}\right]
$$

Now the product of both matrices $F$ and $V^{-1}$ gives

$$
F V^{-1}=\left[\begin{array}{ccc}
\frac{(\beta \lambda)\left(\alpha \mu-P_{2}\right)+\beta \lambda \alpha}{(\lambda+\theta+\mu)\left(\alpha+\mu-P_{1}\right)\left(\gamma+\mu-P_{2}\right)} & \frac{\beta\left(\gamma+\mu-P_{2}\right)+\beta \alpha}{\left(\alpha+\mu-P_{1}\right)\left(\gamma+\mu-P_{2}\right)} & \frac{\beta}{\gamma+\mu-P_{2}} \\
0 & 0 & 0 \\
0 & 0 & 0
\end{array}\right]
$$

The eigenvalues of $F V^{-1}$ are the solutions of the characteristic equation $\left|F V^{-1}-I k\right|=0$. Here $I$ is an identity matrix, $k$ are the eigenvalues of $F V^{-1}$. Thus, the eigenvalues are $k_{1}=\left\{\left[\beta \lambda\left(\alpha \mu-P_{2}\right)+\beta \lambda \alpha\right] /\left[(\lambda+\theta+\mu)\left(\alpha+\mu-P_{1}\right)\left(\gamma+\mu-P_{2}\right)\right]\right\}$, and $k_{2}=k_{3}=0$.

By definition, the dominant eigenvalue is the basic reproduction number $R_{0}$ of the model. In the present case, it is clearly seen to be $k_{1}$. Thus, the reproduction number is given by $R_{0}=\rho\left(F V^{-1}\right)=\left\{\left[\beta \lambda\left(\alpha \mu-P_{2}\right)+\beta \lambda \alpha\right] /\left[(\lambda+\theta+\mu)\left(\alpha+\mu-P_{1}\right)\left(\gamma+\mu-P_{2}\right)\right]\right\}$. 


\subsection{Disease Free Equilibrium Point}

Let $E_{0}=\left(S^{*}, E^{*}, I_{1}{ }^{*}, I_{2}{ }^{*}, R^{*}\right)$ represents the disease free equilibrium point of the modified model $S E I_{1} I_{2} R$. In absence of the infection it happens that $E^{*}=I_{1}{ }^{*}=I_{2}{ }^{*}=0$. Equilibrium points are obtained by setting zero the left hand sides of the equations $(5)-(9)$. Now the other components of the disease free equilibrium point $E_{0}$ satisfy the following equations:

$$
\begin{gathered}
b-(\theta+\mu) \mathrm{S}^{*}+\delta R^{*}=0 \\
\theta \mathrm{S}^{*}-(\mu+\delta) R^{*}=0
\end{gathered}
$$

On solving (14) and (15) the expressions for $S^{*}$ and $R^{*}$ are obtained as

$$
\begin{gathered}
R^{*}=b \theta / \mu(\theta+\mu+\delta) \\
\mathrm{S}^{*}=b(\mu+\delta) / \mu(\theta+\mu+\delta)
\end{gathered}
$$

Therefore, the disease free equilibrium point can be expressed as $E_{0}=\left(\mathrm{S}^{*}, 0,0,0, \mathrm{R}^{*}\right)$. Here in $E_{0}$, the expressions for $S^{*}$ and $R^{*}$ are given by (12) and (13) respectively.

\section{Stability Analysis of the Model}

In this section the stability analysis of the equilibrium points of the model will be considered and made. It has been shown that the disease free equilibrium point is locally and globally stable. the endemic equilibrium point is also identified and formulated.

\subsection{Local Stability Analysis of the Disease Free Equilibrium Point}

To determine the stability of the system at the disease-free equilibrium point, the linearized system of equations about the equilibrium point will be considered.

Theorem 3: The disease free equilibrium point $E_{0}=$ $\left\{\frac{b(\mu+\delta)}{\mu(\theta+\mu+\delta)}, 0,0,0, \frac{b \theta}{\mu(\theta+\mu+\delta)}\right\}$ is locally stable if $R_{0}<1$.

Proof: The Jacobian matrix at the equilibrium point $E_{0}$ can be expressed as

$$
J\left(E_{0}\right)=\left[\begin{array}{c}
\frac{-\beta\left(I_{1}+I_{2}\right)}{N}-(\theta+\mu) \\
\frac{\beta\left(I_{1}+I_{2}\right)}{N} \\
0 \\
0 \\
\theta
\end{array}\right.
$$

$$
\left.\begin{array}{ccc}
\frac{-\beta S}{N} & \frac{-\beta S}{N} & \delta \\
\frac{\beta S}{N} & \frac{\beta S}{N} & 0 \\
P_{1}-(\alpha+\mu) & 0 & \\
\alpha & P_{2}-(\gamma+\mu) & 0 \\
0 & 0 & -(\delta+\mu)
\end{array}\right]
$$

Here for simplicity the notations used include: $u=$ $(\theta+\mu), v=(\lambda+\theta+\mu), w=\left(\alpha+\mu-P_{1}\right), x=$ $\left(\gamma+\mu-P_{2}\right)$ and $y=(\delta+\mu)$. The characteristic equation can be split into two equations as

$$
\begin{gathered}
{[(y+k)(u+k)-\theta \delta]=0} \\
\left|\begin{array}{ccc}
-(v+k) & \beta & \beta \\
\lambda & -(w+k) & 0 \\
0 & \alpha & -(x+k)
\end{array}\right|=0
\end{gathered}
$$

The equation (14) gives two eigenvalues and (15) gives the remaining three values. First of all it is to be shown that the two eigenvalues of (14) are negative. The equation (14) can be expressed in quadratic form as $k^{2}+(\theta+2 \mu+\delta) k+$ $\mu(\theta+\mu+\delta)=0$. The solutions of this quadratic equation are the eigenvalues. The two eigenvalues are real distinct negatives provided that if the (i) coefficient of $k$ and the constant terms are positive (ii) discriminate is positive. The first condition holds true because all the parameters involved here are positive quantities. However the second condition holds true if $(\theta+2 \mu+\delta)^{2}>4(\theta+\mu+\delta)$.

Now it is to be shown that the three eigenvalues of (15) are real distinct and negative. The equation (15) can be expressed in cubic form as $p(k)=k^{3}+k^{2}(v+w+x)+$ $k(v w+v x+w x-\beta \lambda)+v w x\left(1-R_{0}\right)$.

According to Routh - Hurwitz criteria the three roots of a polynomial of order three of the type $P(k)=k^{3}+a_{1} k^{2}+$ 
$a_{2} k+a_{3}$ are real distinct negatives if the coefficients satisfy the conditions $a_{1}>0, a_{2}>0$ and $a_{1} a_{2}>a_{3}$. It is straight forward to verify that these conditions are satisfied and hence the last three eigenvalues of the Jacobian matrix are also real distinct and negatives.

Clearly it can be observed that the first two conditions of Routh - Hurwitz are satisfied in case of $p(k)$ and the third condition is satisfied provided that $(v+w+x)(v w+$ $v x+w x-\beta \lambda)>v w x\left(1-R_{0}\right)$.

Therefore the disease free equilibrium point of system of ordinary differential equations (5) to (9) is locally asymptotically stable if $R_{0}<1$ as long as the following two conditions on the parameters hold good:

$$
(\theta+2 \mu+\delta)^{2}>4(\theta+\mu+\delta)
$$

$$
(v+w+x)(v w+v x+w x-\beta \lambda)>v w x\left(1-R_{0}\right)
$$

\subsection{Global Stability of the Disease Free Equilibrium Point}

Theorem 4: The disease free equilibrium point $E_{0}=$ $\left\{\frac{b(\mu+\delta)}{\mu(\theta+\mu+\delta)}, 0,0,0, \frac{b \theta}{\mu(\theta+\mu+\delta)}\right\}$ is globally stable if $R_{0}<1$.

Proof: By the comparison theorem, the rate of change of the variables representing the infected components of the model system of differential equations (5) - (9) can be rewritten as

$$
\left[\begin{array}{l}
\frac{d E}{d t} \\
\frac{d I_{1}}{d t} \\
\frac{d I_{2}}{d t}
\end{array}\right]=[F-V]\left[\begin{array}{l}
E(t) \\
I_{1}(t) \\
I_{1}(t)
\end{array}\right]-\beta\left(I_{1}+I_{2}\right)\left[\begin{array}{c}
1-\frac{S}{N} \\
0 \\
0
\end{array}\right]
$$

Note that $(S / N)=1$ holds good at the disease free equilibrium point $E_{0}$. Thus, the above matrix equation reduces to

$$
\begin{aligned}
& {\left[\begin{array}{c}
\frac{d E}{d t} \\
\frac{d I_{1}}{d t} \\
\frac{d I_{2}}{d t}
\end{array}\right]=[F-V]\left[\begin{array}{l}
E(t) \\
I_{1}(t) \\
I_{1}(t)
\end{array}\right]} \\
& S^{* *}=\left\{N(\lambda+\theta+\mu)\left(\alpha+\mu-P_{1}\right)\left(\gamma+\mu-P_{2}\right)\right\} /\left\{\beta \lambda\left(\gamma+\mu-P_{2}+\alpha\right)\right\} \\
& E^{* *}=\frac{\left(\alpha+\mu-P_{1}\right)\left[N(\delta+\mu)(\theta+\mu)-\theta \delta N-b R_{0}(\delta+\mu)\right]\left[\lambda R_{0}\left(\gamma+\mu-P_{2}\right)(\delta+\mu)\right]}{\lambda(\delta+\mu) R_{0}\left[R_{0} \delta \theta\left(\gamma+\mu-P_{2}\right)\left(\alpha+\mu-P_{1}\right)-\beta \lambda(\delta+\mu)\left(\gamma+\mu-P_{2}+\alpha\right)\right]} \\
& I_{1}{ }^{* *}=\frac{\left[N(\delta+\mu)(\theta+\mu)-\theta \delta N-b R_{0}(\delta+\mu)\right]\left[\lambda R_{0}\left(\gamma+\mu-P_{2}\right)(\delta+\mu)\right]}{R_{0}(\delta+\mu)\left[R_{0} \delta \theta\left(\gamma+\mu-P_{2}\right)\left(\alpha+\mu-P_{1}\right)-\beta \lambda(\delta+\mu)\left(\gamma+\mu-P_{2}+\alpha\right)\right]} \\
& \mathrm{I}_{2}{ }^{* *}=\left\{\frac{\alpha R_{0}\left[N(\delta+\mu)(\theta+\mu)-\theta \delta N-b R_{0}(\delta+\mu)\right]\left[\lambda R_{0}(\delta+\mu)\right]}{(\delta+\mu)\left[R_{0} \delta \theta\left(\gamma+\mu-P_{2}\right)\left(\alpha+\mu-P_{1}\right)-\beta \lambda(\delta+\mu)\left(\gamma+\mu-P_{2}+\alpha\right)\right]}\right\} \\
& R^{* *}=\frac{\theta}{(\delta+\mu)}\left\{\frac{N}{R_{0}}+\frac{\left(\alpha+\mu-P_{1}\right)\left[(\delta+\mu)(\theta+\mu) N-\theta \delta N-b R_{0}(\delta+\mu)\right]\left[\left(\gamma+\mu-P_{2}\right)(\delta+\mu) \lambda R_{0}\right]}{\lambda(\delta+\mu) R_{0}\left[\left(\gamma+\mu-P_{2}\right) R_{0} \delta \theta\left(\alpha+\mu-P_{1}\right)-\beta \lambda(\delta+\mu)\left(\gamma+\mu-P_{2}+\alpha\right)\right]}\right\}
\end{aligned}
$$

It has been shown that matrix $[F-V]$ in (18) whose eigenvalues are located on its main diagonal which real and negative. It follows that the linearized differential inequality system (18), is stable whenever $R_{0}<1$. Consequently $\left(E, I_{1}, I_{2}\right)=(0,0,0)$ as $t \rightarrow \infty$ and evaluating the system of differential equation (5)-(9) at $\left(E=I_{1}=I_{2}=0\right)$ gives $E_{0}=\{(b(\mu+\delta)) /(\mu(\theta+$ $\mu+\delta)), 0,0,0, b \theta /(\mu(\theta+\mu+\delta))\}$. Thus $\left(S^{*}, E^{*}, I_{1}{ }^{*}, I_{2}{ }^{*}\right) \rightarrow E_{0}$ and also as $t \rightarrow \infty$ the condition $R_{0}<1$ implies that the disease free equilibrium point is globally asymptotically stable.[15]

\subsection{The Endemic Equilibrium Point of Modified Model}

Endemic equilibrium are steady state solutions where the disease persist in the population i.e., all the state variables are positive. The rabies infection will persist in the population and the endemic equilibrium of the modified model is given 要 $E_{1}=\left(S^{* *}, E^{* *}, I_{1}{ }^{* *}, I_{2}{ }^{* *}, R^{* *}\right)$. At this equilibrium point $E_{1}$ the right hand sides of the model differential equations
Theorem 5 The positive endemic equilibrium point $E_{1}$ of the system of equations (5) to (9) is locally asymptotically stable if $R_{0}>1$.

Proof To determine the stability of the system at the endemic equilibrium point $E_{1}$, the linearized system of equations (5) to (9) about the equilibrium point will be considered. Its Jacobian matrix can be constructed as

$$
J\left(E_{1}\right)=\left[\begin{array}{cccccc}
-u & 0 & -\beta S^{* *} & -\beta S^{* *} & \delta \\
\beta I^{* *} & -v & \beta S^{* *} & \beta S^{* *} & 0 \\
0 & \lambda & -w & 0 & 0 \\
0 & 0 & \alpha & -x & 0 \\
\theta & \theta & 0 & 0 & -y &
\end{array}\right]
$$

Here the notations used are: $u=\left(\beta\left(I_{1}{ }^{* *}+I_{2}{ }^{* *}\right) S^{* *}\right) / N-$ $(\theta+\mu) S^{* *} ; \mathrm{v}=(\lambda+\theta+\mu) ; \mathrm{w}=\left(\alpha+\mu-P_{1}\right) ; x=$ $\left(\gamma+\mu-P_{2}\right) ; \mathrm{y}=(\delta+\mu) ; \beta I^{* *}=$ 
$\beta\left(I_{1}{ }^{* *}+I_{2}{ }^{* *}\right) / N$; and $\beta S^{* *}=\beta S^{* *} / N$. Now the characteristic polynomial of the Jacobian matrix $\mid J\left(E_{1}\right)-$ $k I \mid=0$, where $k$ is the eigenvalues and $I$ is an identity matrix of class five, takes the form as

$$
P(k)=k^{5}+B_{1} k^{4}+B_{2} k^{3}+B_{3} k^{2}+B_{4} k+B_{5}
$$

Here in (19), some notations have been used to represent the following expressions:

$$
\begin{gathered}
B_{1}=y+u+v+w+x \\
B_{2}=y u+y v y w+y x+u v+u w+u x+v w \\
+v x+w x-\lambda \beta S^{* *}-\delta \theta \\
B_{3}=y u v+y u w+y u x+y v w+y v x+y w x+u v w \\
+u v x+u w x+v w x+\beta^{2} I^{* *} S^{* *} \\
-\lambda \beta S^{* *}(x+\alpha+y+u) \\
-\delta \theta(v+w+x) \\
B_{4}=y u v w+y u v x+y u w x+y v w x+u v w x \\
+\beta I^{* *} S^{* *}(x+y+\alpha)+\delta \theta \lambda \beta S^{* *} \\
-\lambda \beta S^{* *}(y(x+\alpha)+u(x+\alpha)+y u) \\
-\delta \theta \beta I^{* *}(x+w)-\delta \theta(v w+v x \\
+w x) \\
+\beta^{2} I^{* *} S^{* *} y(x+\alpha)+\delta \theta \lambda \beta S^{* *}(x+\alpha)
\end{gathered}
$$

By Routh - Hurwitz criteria the determinant of Hurwitz matrix becomes positive if the following conditions hold true: $B_{1}>0, B_{2}>0, B_{3}>0, B_{4}>0, B_{5}>0, B_{3} B_{4}>$ $B_{2} B_{5}, B_{5} B_{1} B_{2}>B_{3}, B_{5}>B_{1} B_{4}$. All the parameters of the present model are positive. Therefore, (i) $B_{2}>0$ if and only if $y u+y v y w+y x+u v+u w+u x+v w+$ $v x+w x>\lambda \beta S^{* *}+\delta \theta$ (ii) $B_{3}>0$ If and only if $\quad y u v+y u w+y u x+y v w+y v x+y w x+u v w+$ $u v x+u w x+v w x+\beta^{2} I^{* *} S^{* *}>\lambda \beta S^{* *}(x+\alpha+u)+$ $\delta \theta(v+w+x)$ (iii) $B_{4}>0$ if and only if yuvw + $y u v x+y u w x+y v w x+u v w x+\beta^{2} I^{* *} \lambda S^{* *}(x+y+$ $\alpha)+\delta \theta \lambda \beta S^{* *}>\lambda \beta S^{* *}(y(x+\alpha)+u(x+\alpha)+$ $y u)+\delta \theta \beta I^{* *}(x+w)+\delta \theta(v w+v x+w x)$

$B_{5}>0$ If and only if yuvx $+\beta^{2} \lambda S^{* *} I^{* *} y(x+\alpha)>$ $\lambda \beta S^{* *} y u(x+\alpha)+\delta \theta v w x\left(\beta I^{* *}+v\right)$

When all the conditions for $B_{1}, B_{2}, B_{3}, B_{4}, B_{5}$ hold and , $B_{1} B_{2} B_{3}>B_{3}{ }^{2}, \mathrm{~B} \quad\left(B_{1} B_{2} B_{3}>B_{3}{ }^{2}+B_{1}{ }^{2} B_{4}\right.$ and $\left(B_{1} B_{4}-B_{5}\right)\left(B_{1} B_{2} B_{3}-B_{3}{ }^{2}-B_{1}{ }^{2} B_{4}\right)>B_{5}\left(B_{1}+\right.$ $\left.B_{2}-B_{3}\right)^{2}+B_{1} B_{5}{ }^{2}$ holds. Hence all the roots of the characteristic polynomial (19) are negative this verify that the system (5) to (9) is locally asymptotically stable if $R_{0}>1$.

\section{Numerical Simulations}

Numerical simulations of the model have been carried out using MATLAB inbuilt function ode 45; using the RungeKutta of order four. The main focus of the simulation is to investigate the response of model parameters for rabies epidemic. In simulating the $\mathrm{S} E \mathrm{I}_{1} \mathrm{I}_{2} \mathrm{R}$ model equations (5) (9) representing the transmission of rabies disease among dogs with vaccination the following parametric values selected based on assumptions are used: $\beta=0.0198 ; \lambda=$
$0.079 ; \gamma=0.089 ; \theta=0.5 ; \delta=0.09824 ; \mu=$

0.0292; $\alpha=0.0591 ; b=0.9897 ; P_{1}=P_{2}=0.02$. Each of these values are numbers per year. The simulation graphs are given as figures.

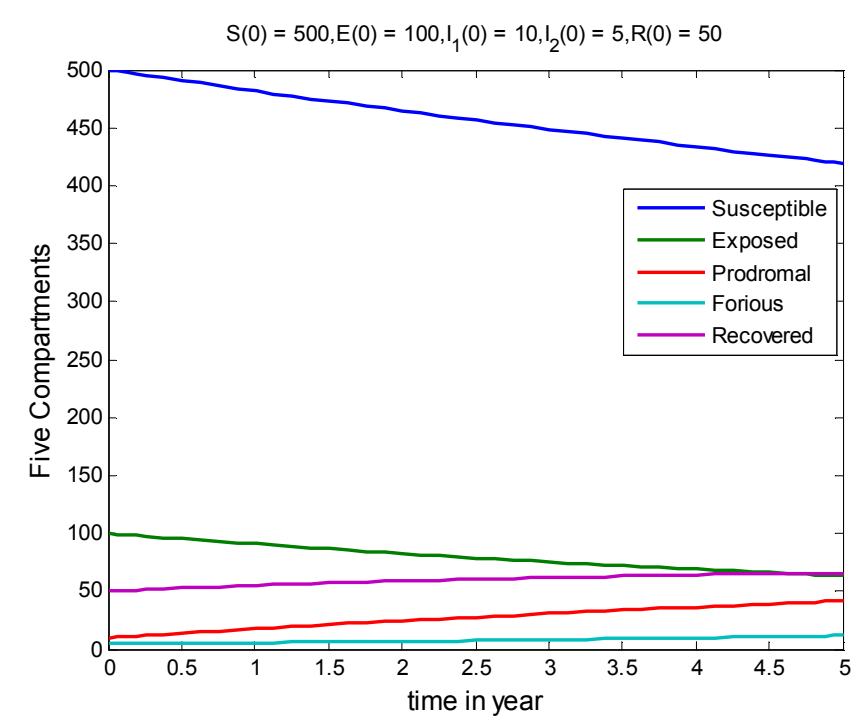

Figure 3. Numerical simulation of dog rabies with $\theta=0.02$ and $P_{1}=P_{2}=$ 0.09 .

In Figure 3 it can be observed that the population sizes of the compartments (i) $S$ and $E$ decrease while (ii) that of $I_{1}, I_{2}$ and $R$ increase. However, the variations are of slower rates. Thus, the simmulation reveals that the infection spreads and the result will be an epedemic. But the spread of the epedemic is slower.

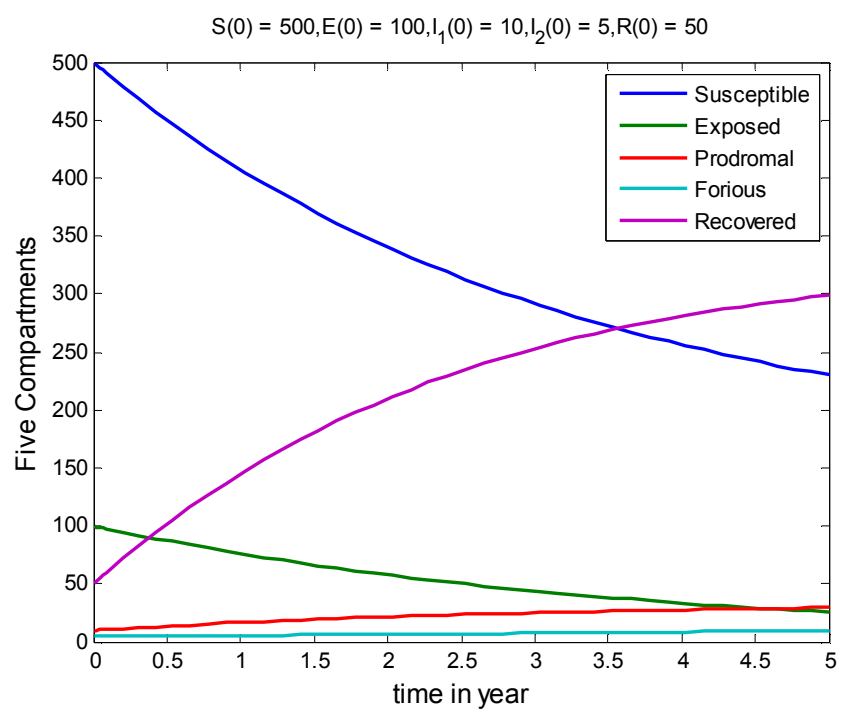

Figure 4. Numerical simulation of $\operatorname{dog}$ rabies with $\theta=0.2$ and $P_{1}=P_{2}=$ 0.07 .

In Figure 4 it can be observed that the population sizes of the compartments (i) $S$ and $E$ decrease while (ii) that of $I_{1}, I_{2}$ and $R$ increase. However, the variations are of faster rates. Thus, the simmulation reveals that the infection spreads and the result will be an epedemic. But the spread of the epedemic is faster. 


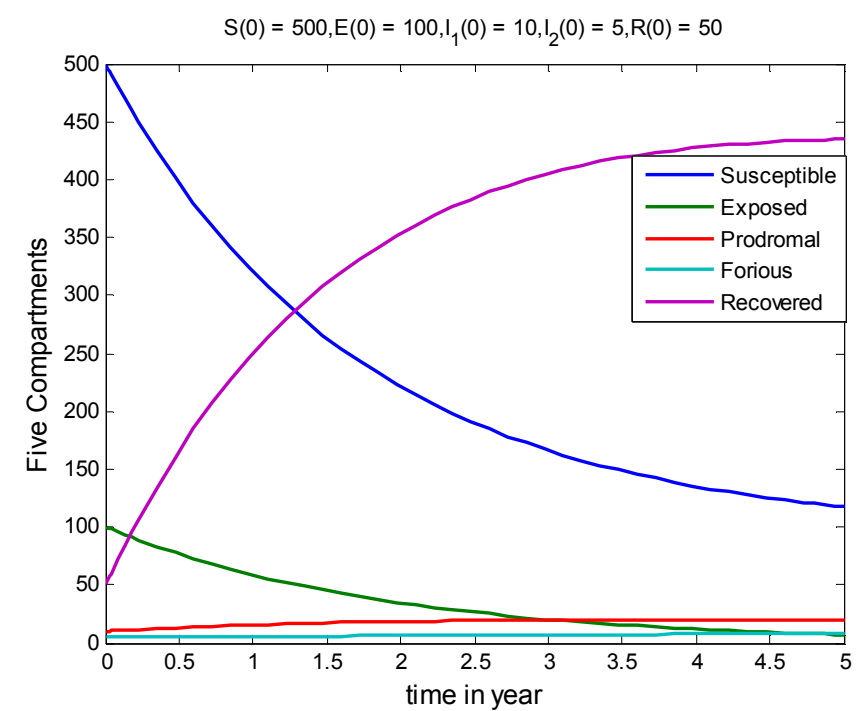

Figure 5. Numerical simulation of dog rabies with $\theta=0.46$ and $P_{1}=P_{2}=$ 0.05 .

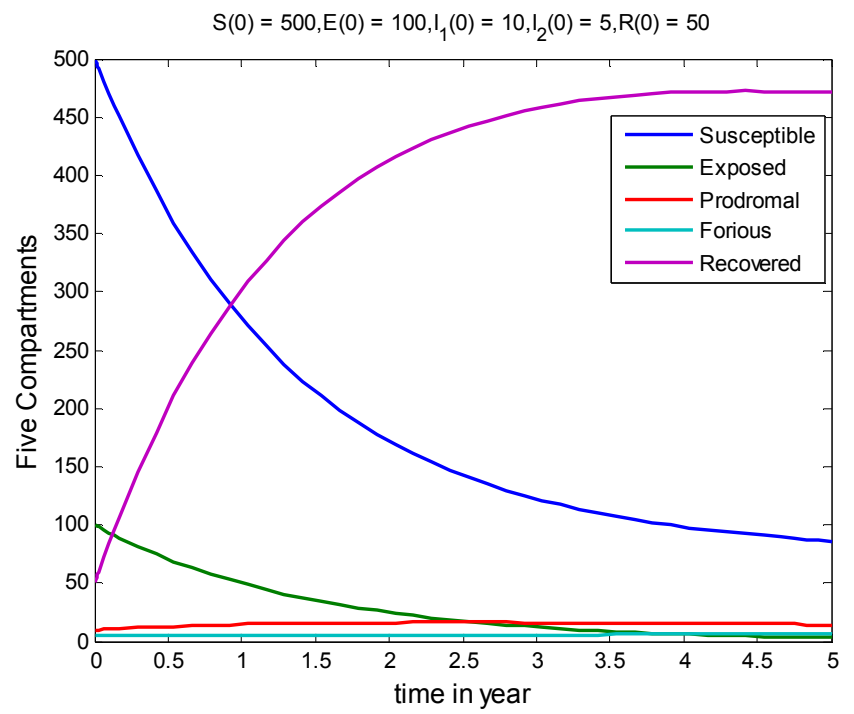

Figure 6. Numerical simulation of dog rabies with $\theta=0.62$ and $P_{1}=$ $P_{2}=0$.

In figures 5 and 6 the population dynamics of $S E I_{1} I_{2} R$ epidemic compartmental model with deffirent values of vaccination rates $\theta$ and immigration rates $P_{1}$ and $P_{2}$ are simulated. The susceptible and exposed dogs decrease. The number of prodromal and forious stage dogs are slowly decreasing to zero. The number of recoverd stage dogs increases when times. It can be concluded from these simulated graphs that as vaccination rate increases and immigration rate decreases the epidemic seems dies out.

Some special and main features of the present model are: (i) The total dog population is divided into five compartments; (ii) the model has $S E I_{1} I_{2} R$ structure; (iii) Vaccination is considered; (iv) infective immigration of the dog population is considered (v) The dogs mix heterogeneously; and (vi) reproductive number depends on many parameters.

\section{Conclusions}

In this paper, new mathematical model is proposed to study the impact of infective immigrants on the spread of dog rabies in a variable size population with a constant recruitment of susceptible. Stability analysis and numerical simulations on the model shows that the disease dies out if the immigration of the infected dogs is controlled, the vaccination and the treatments are improved. Otherwise, the disease spreads rapidly in the dog population and it will become an epidemic. Also certain model parameters such as contact rate could increase the spread of the disease.

\section{References}

[1] Tesfaye Tadesse Ega, Living stone S. Luboobi, Modeling the Dynamics of Rabies Transmission with Vaccination and Stability Analysis, Applied and Computational Mathematics, (2015), 4 (6): 409-419.

[2] John Bingham, Canine Rabies Ecology in Southern Africa, Emerging Infectious.www.cdc.gov/eid. vol. 11, No. 9, September 2005.

[3] Elif Demisrci, A New Mathematical Approach for Rabies Endemic, Applied Mathematical Sciences, Vol. 8, (2015), no. 2, 59-67.

[4] Kwaku Marri Addo. An SEIR Mathematical Model for Dog Rabies. Case Study: Bongo District, Ghana, M.Sc. Dissertation Kwame Nkrumah University of science and Technology, 2012.

[5] Sandra DW, A. Arsum Arsin, Anwar Mallongi, The Dynamic Model Approach in Estimating Rabies Death in North Toroja Regency, International Journal of Sciences: Basic and Applied Research (IJSAR)(2015) volume 24, No 1. Pp 421-429.

[6] Tiffany Ngo Leung, Mathematical Models for dog rabies that include the curtailing effect of human intervention, Australian Mathematical Sciences institute.

[7] Demsis Dejene, Purnachandra Rao Koya, Population Dynamics of Dog Rabies Disease, IOSR Journal of Mathematics (IOSRJM) volume 12, Issue 3 ver. IV (may.-Jun 2016), pp 110-120.

[8] Bernoulli, D. Blower, S. (2004). An attempt at a new analysis of the mortality caused by smallpox and of the advantages of inoculation to prevent it. Reviews in Medical Virology, 14, $275 \mathrm{a} " 288$.

[9] Dancho Desaleng, Purnachandra Rao Koya. The Role of Polluted Air and Population Density in the Spread of Mycobacterium Tuberculosis Disease, Journal of Multidisciplinary Engineering Science and Technology (JMEST). Vol. 2, Issue 5, May - 2015, Pp 1212 - 20. ISSN: $3159 \quad-\quad 0040 . \quad$ http://www.jmest.org/wp content/uploads/JMESTN42350782.pdf

[10] Ethiopian health and nutrition research institute (EHNRI). Rabies case report 1990 - 2010, EHNRI, Addis Ababa, Ethiopia, 2011. 
[11] Purnachandra Rao Koya and Dejen Ketema Mamo. Ebola Epidemic Disease: Modeling, Stability Analysis, Spread Control Technique, Simulation Study and Data Fitting. Journal of Multidisciplinary Engineering Science and Technology (JMEST), Vol. 2, Issue 3, March 2015, pp 476 - 84. ISSN: $3159-0040$. http://www.jmest.org/wpcontent/uploads/JMESTN42350548.p df

[12] S. N. Sivanandam and S. N. Deepa. Linear system design using Routh column polynomials, Songklanakarin J. Sci. Technol. 2007, 29 (6), 1651 - 1659.

[13] F. Brauer and P. van den Driessche 2001 Models for transmission of disease with immigration of infectives, Math. Biosci, 171 (2): 143-1542.

[14] Coffee Megan, Lurie Mark N., and Garnett Geoff P. Modeling the impact of migration on the HIV epidemic in South Africa. AIDS, 21 (3): 343-350, 2007.

[15] Tadele Degefa Bedada, Mihretu Nigatu Lemma and Purnachandra Rao Koya. Mathematical Modeling and simulation study of Influenza disease. Journal of
Multidisciplinary Engineering Science and Technology (JMEST), Vol. 2, Issue 11, November 2015, Pp 3263 - 69. ISSN: 3159 - 0040. http://www.jmest.org/wpcontent/uploads/JMESTN42351208.pdf

[16] Tadele Tesfa Tegegne, Purnachandra Rao Koya and Temesgen Tibebu Mekonnen. Impact of Heterosexuality and Homosexuality on the transmission and dynamics of HIV/AIDS, IOSR Journal of Mathematics (IOSR-JM) e-ISSN: 2278-5728, p-ISSN: 2319-765X. Volume 12, Issue 6 Ver. V (Nov. - Dec. 2016), PP 38-49. DOI: 10.9790/57281206053849 .

[17] Fekadu Tadege Kobe, Purnachandra Rao Koya. Controlling the Spread of Malaria Disease Using Intervention Strategies, Journal of Multidisciplinary Engineering Science and Technology (JMEST), Vol. 2, Issue 5, May 2015, pp $1068-$ 74. ISSN: $3159-0040 . \quad$ http://www.jmest.org/wpcontent/uploads/JMESTN42350745.pdf

[18] Eti DWI Wiraningsih, Folashade Agusto, Lina Aryati. A Stability Analysis of Rabies Model with Vaccination Effect and Culling in Dogs, Applied Mathematical Sc. Vol. (9): 38053817,2015 . 